Recuperación de trabajos de fin de grado

\title{
Identificación de víctimas por parte del Comité Municipal de Lucha Contra la Trata de Personas de Bucaramanga.
}

\section{Análisis de caso}

Victims identification by the Bucaramanga's Municipal Committee Against Human Trafficking. A case analysis

Cristian David Sandoval Bueno 


\section{Resumen}

El presente artículo expone los resultados de un proyecto de investigación que indagó por los procesos de identificación de víctimas de trata de personas por parte del Comité Municipal de Lucha Contra la Trata de Personas de Bucaramanga, Colombia , con el objetivo principal de conocer los procesos de identificación de las mismas. Para dar cumplimiento a este último, se realizó un estudio de caso múltiple. Entre los hallazgos principales se encontró que la identificación de víctimas se compone de tres momentos: recepción del caso, análisis del caso y activación de ruta de asistencia. Sobre la recepción del caso, esta suele ser de forma pasiva, es decir, a través de denuncias ciudadanas o víctimas remitidas por otras instituciones. Respecto al análisis de casos y activación de ruta de asistencia, están centralizadas en las labores administrativas de la secretaría técnica del comité. En síntesis, los procesos de identificación de víctimas de trata parecen ser más procesos administrativos de construcción de caso en los cuales lo más importante son las labores de papeleo para la identificación de una presunta víctima.

Palabras clave

Trata de personas, Identificación, Víctimas, Caso.

\section{Abstract}

This article presents the results of a research Project that investigated the identification processes of human trafficking's victims by the Bucaramanga's Municipal Committe to Fight Human Trafficking. The main objective was to know the processes of victims identification by the Municipal Committee to Combat Human Trafficking of Bucaramanga. To comply with the latter, the research carried out a case analysis. Among the main results, it was found that the victim's identification is compound of three moments: case reception, case analysis and the activation of the assistance route. Regarding the case reception, this is usually passive, that is, through citizen complaints or victims sent by other institutions. Regarding case analysis and assistance route, these are centralized in the functions of the committee's technical secretariat. In summary, the victim's identification processes seems to be more administrative processes of "case construction" in which the most important task is the "paperwork" for the identification of an alleged victim.

\section{Keywords}

Human trafficking, Identification, Victims, Cases. 


\section{Introducción}

La trata de personas es una violación a los derechos humanos que coarta la autonomía de sus víctimas y degrada su dignidad. De acuerdo con el Protocolo para perseguir, reprimir y sancionar la trata de personas, especialmente en mujeres y niños (en adelante, Protocolo de Palermo), la trata consiste en:

(...) la captación, el transporte, el traslado, la acogida o la recepción de personas, recurriendo a la amenaza o al uso de la fuerza u otras formas de coacción, al rapto, al fraude, al engaño, al abuso de poder o de una situación de vulnerabilidad o a la concesión o recepción de pagos o beneficios para obtener el consentimiento de una persona que tenga autoridad sobre otra, con fines de explotación (Naciones Unidas, 2000:2).

Las víctimas de este delito varían según la fuente. De acuerdo con la Counter Trafficking Data Collaborative ${ }^{1}$, desde 2002 hasta septiembre del 2020, a nivel mundial, se han identificado 108.613 víctimas de trata en 164 países (Organización Internacional para las Migraciones, 2020). Por su parte, la Oficina de las Naciones Unidas Contra la Droga y el Delito (en adelante, UNODC) (2018) reporta, desde 2003², alrededor de 225.000 víctimas en el mundo. De 2.949 casos detectados en Sudamérica, en el 58\% hubo finalidad de explotación sexual, en el $32 \%$ trabajo forzado y en un $10 \%$ de los casos no está claro el tipo de explotación.

En esta línea, el Departamento de Estado de los Estados Unidos ha manifestado que en el año 2019 en Colombia se detectaron 124 víctimas. De ellas, 81 sufrieron explotación sexual, 6 trabajo forzado, 11

1 Base de datos lanzada en noviembre de 2017 en la cual confluye información de organizaciones (oficiales y civiles) de todo el mundo, entre ellas la OIM, Polaris y Liberty Shared. Las cifras de esta base de datos, hasta febrero de 2020, abarcan 172 países de explotación y 169 nacionalidades de todos los sobrevivientes de trata de personas reportados.

2 Este reporte incluye información desde el 2003 ya que en dicho año entra en plena vigencia el Protocolo de Palermo. Para su construcción se cuenta con datos de 142 países y se abarca más del 94\% de la población mundial (UNODC, 2018). 
fueron explotadas en servicios domésticos y 12 de matrimonio servil, mientras que para 14 casos se desconoce la finalidad de explotación (Department of State, 2020). De acuerdo con el Ministerio del Interior Nacional (2020), desde el 2013 al 2019 se han asistido en Colombia 610 víctimas de trata de personas.

Por su parte, en Santander se han atendido entre 2013 y 2019 a 18 víctimas: 16 de trata externa explotadas en países extranjeros y 2 de trata interna explotadas en Colombia (Ministerio del Interior, 2020). Según el Sistema Penal Oral Acusatorio (en adelante, SPOA) de la Fiscalía General de la Nación, entre 2010 y 2016, hubo 57 noticias criminales por el delito de trata de personas. En el $56 \%$ de los casos las víctimas fueron mujeres, $23 \%$ fueron hombres y en un $21 \%$ de los casos no se reporta el sexo de la víctima (UNODC y Gobernación de Santander, 2018). En la capital del departamento, la policía reportó 15 casos de trata entre los años 2010 y 2016 . Entre ellos, el $80 \%$ fueron mujeres y el 20\% hombres (UNODC y Gobernación de Santander, 2018).

Finalmente, en el año 2010 se creó el Comité Municipal de Lucha contra la Trata de Personas de Bucaramanga mediante el acuerdo municipal 017. Mientras que en Colombia el comité de Santander se ha destacado por ser uno de los más activos (Aceros, Vargas y Reyes 2017), el comité de Bucaramanga es el que más víctimas ha asistido en los últimos años en el departamento. Según datos del Comité Departamental de Lucha contra la Trata de Personas de Santander, desde 2016 han atendido 4 víctimas (2019).

En este contexto, la presente investigación tuvo como propósito darle contenido a un campo de investigación poco nutrido en Colombia y Bucaramanga: asistencia a víctimas de trata de personas. La exploración documental dejó en evidencia que los procesos operatorios/ administrativos de asistencia a sobrevivientes de este delito no han sido extensamente estudiados en el país. Por lo tanto, este proyecto se planteó como pregunta directriz: ¿cómo son las dinámicas institucionales para la asistencia y protección a víctimas de trata de personas por parte del comité de lucha contra la trata de personas de Bucaramanga? 
De esta manera, el presente artículo expone los resultados de uno de los objetivos específicos: conocer los procesos de identificación de víctimas por parte del Comité Municipal de Lucha Contra la Trata de Personas de Bucaramanga, ya que fue la categoría más saturada en información cualitativa y cuantitantivamente.

\section{Antecedentes}

En primer lugar, cabe destacar que esta investigación tuvo un alcance local/municipal, por lo cual la revisión documental se enfocó en investigaciones que abordan la identificación de víctimas de trata en Colombia. Luego de esta criba, hay dos documentos a discutir relacionados con identificación de víctimas.

El primero fue escrito por Aceros, Vargas y Reyes (2017) y titulado Trayectorias territoriales de la asistencia a víctimas de trata de personas. Análisis cualitativo desde la perspectiva de los actores institucionales de Santander, Colombia. De sus hallazgos, se destaca un conjunto de buenas prácticas y debilidades de los dispositivos de asistencia y protección en el departamento. Entre las buenas prácticas los autores resaltan: la prontitud con la cual los comités locales identificaron la situación de trata y activaron la ruta de atención para iniciar el proceso de asistencia y protección; la forma en que los funcionarios abordaron las primeras entrevistas o encuentros con las víctimas; y la cooperación entre instituciones del Estado y organizaciones no gubernamentales. Por su parte, entre las debilidades, mencionan cinco tipos: poco conocimiento de funcionarios públicos sobre la dinámica de la trata de personas; dificultades en la coordinación interinstitucional, particularmente entre instituciones de un mismo comité -municipal o departamental- y entre entidades territoriales y el nivel nacional, es decir, instituciones o instancias de instituciones que hacen parte del Comité Interinstitucional de Lucha Contra la Trata; recursos humanos y materiales limitados; poca operatividad de muchos comités municipales; y la falta de seguimiento a la asistencia brindada a víctimas.

El otro documento es el trabajo de Alarcón (2016) titulado Escenario para la asistencia y protección a víctimas de trata de personas en Colombia. Allí, a través de una revisión documental y entrevistas 
semiestructuradas a expertos en trata de personas, y un sobreviviente de este delito, se establecen los avances del Comité Distrital de Lucha Contra la Trata de Personas de Bogotá. El autor encuentra, como un punto a mejorar, que los funcionarios encargados cambian con regularidad en las instituciones, lo cual dificulta los procesos de protección a víctimas. Por otro lado, según los actores de la sociedad civil, los enfoques de acción planteados por la Estrategia Nacional de Lucha Contra la Trata de Personas no son plenamente integrados en las prácticas institucionales.

De esta manera, ambas investigaciones apuntan a hallazgos de tipo procedimental y estructural. En el apartado estructural, se encuentran particularidades como el poco recurso humano, técnico y económico para llevar adelante procesos de investigación y para la prestación de servicios de asistencia a víctimas. De igual forma se hace presente el desconocimiento de las particularidades del delito por algunos funcionarios sobre los cuales recae la responsabilidad de identificar y restablecer derechos de las víctimas sobrevivientes. Asimismo, los procesos de asistencia se ven afectados por algunas carencias procedimentales, esto se hace evidente en el constante cambio de funcionarios públicos a cargo de atender y darle seguimiento al delito, así como una limitada implementación práctica de las recomendaciones establecidas en la Estrategia Nacional de Lucha Contra la Trata.

Como es visible, aunque existen esfuerzos aislados por entender los procesos de identificación y asistencia a víctimas en Colombia, aún no han sido debidamente contrastados y/o sistematizados de manera que se constituya un cuerpo teórico o descriptivo/hermenéutico que dé cuenta de ellos.

\section{Metodología}

El apartado anterior expresa la ausencia de un marco teórico que dé cuenta sobre los procesos de asistencia y protección a víctimas de trata de personas. Por ello, se realizó un estudio de caso múltiple en el que se examinaron procesos de asistencia y protección a víctimas de trata de personas coordinados por el Comité Municipal de Bucara- 
manga de los últimos 3 años, lo cual permitió reconstruir cada uno de ellos para su posterior análisis en conjunto.

De acuerdo con Yin (2014), un estudio de casos se centra en la manifestación de un fenómeno en un espacio-tiempo determinado y, desde una perspectiva integradora, aborda variados fenómenos asociados que pueden ir desde el comportamiento individual o de pequeños grupos, a procesos organizativos y gerenciales, relaciones internacionales, etc. En este sentido, un caso es la manifestación de un fenómeno social y la forma en que afecta a un individuo, grupo de personas, instituciones u otros colectivos, en un momento y lugar específicos. El estudio de caso es apropiado en investigaciones cuyas preguntas indaguen por el cómo y el porqué de fenómenos poco explorados (Yin, 2014). Además, suele recomendarse para construir teoría emergente (Chetty, 2016).

Por todo lo anterior, se ha reconocido que los estudios de caso son compatibles con la Teoría Fundamentada en la medida que esta suele partir de casos individuales a partir de los cuales se puedan generar estudios comparativos (Strauss y Corbin, 2002; Halaweh, Fibler y McRobb, 2008). Es por esto que parte de su proceso metodológico ha sido usado en este proyecto, tal como se expondrá más adelante.

Para este proyecto, los casos son los procesos de asistencia y protección a víctimas de trata de personas liderados por el comité municipal de Bucaramanga desde el 2016 hasta el 2019. Los casos analizados fueron tres en total. Algunos fueron reconocidos y atendidos, en primera instancia, por el Comité Distrital de Lucha Contra la Trata de Personas de Bogotá y por el Ministerio del Interior Nacional. Además, fueron remitidos a Bucaramanga para continuar con la ruta iniciada en Bogotá D.C.

Tabla 1: casos de trata de personas atendidos por el comité de Bucaramanga.

\begin{tabular}{|l|l|c|c|c|}
\hline $\begin{array}{c}\text { Víctima } \\
\text { (pseudónimo) }\end{array}$ & Tipo de trata & $\begin{array}{c}\text { Finalidad de } \\
\text { explotación } \\
\text { Andrés }\end{array}$ & $\begin{array}{c}\text { Trata interna } \\
\text { vosible de la } \\
\text { vima }\end{array}$ & $\begin{array}{c}\text { Tipo de asistencia } \\
\text { prestada }\end{array}$ \\
\hline Daniela & Trata interna & Hombre & Asistencia mediata \\
\hline Camila & Trata externa & $\begin{array}{c}\text { No se especifica. } \\
\text { Posible explotación } \\
\text { sexual }\end{array}$ & Mujer & $\begin{array}{c}\text { Asistencia inmediata } \\
\text { - asistencia mediata }\end{array}$ \\
\hline & \begin{tabular}{c} 
Asistencia mediata \\
\hline
\end{tabular} & & \\
\hline
\end{tabular}


Por su parte, para la obtención de información se contó con la participación de cuatro funcionarias públicas, dos de las cuáles eran funcionarias de la Alcaldía de Bucaramanga y dos de la Gobernación de Santander que tuvieron responsabilidades en la atención a las víctimas $^{3}$ y una ex funcionaria de una institución que atiende a víctimas de trata de personas en Bucaramanga. Adicionalmente, se contó con la participación de una víctima de trata que fue sujeto de asistencia y protección.

Tabla 2: participantes de la investigación.

\begin{tabular}{lcc}
\hline Participante & Rol en la ruta de asistencia & Pseudónimo \\
\hline 1 & $\begin{array}{c}\text { Secretaría técnica comité municipal de } \\
\text { Bucaramanga }\end{array}$ & Marta \\
3 & $\begin{array}{c}\text { Profesional en psicología de la oficina de la } \\
\text { mujer de la alcaldia de Bucaramanga } \\
\text { Funcionaria Grupo de Paz y DD. HH de la } \\
\text { gobernación de Santander }\end{array}$ & Alejandra \\
4 & $\begin{array}{c}\text { Funcionaria Grupo de Paz y D. HH de la } \\
\text { gobernación de Santander }\end{array}$ & Mónica \\
5 & $\begin{array}{c}\text { Ex funcionaria del Servicio Nacional de } \\
\text { Aprendizaje (SENA) }\end{array}$ & Patricia \\
& Sobreviviente de trata de personas asistida por \\
el Comité de Bucaramanga. & Daniela \\
\hline
\end{tabular}

Los participantes fueron seleccionados a través de un muestreo de casos-tipo. De acuerdo con Hernández, Fernández y Baptista (2010), este tipo de muestra es apropiada en investigaciones cuyo objetivo apunta a la calidad y profundidad de la información en lugar de la cantidad y estandarización. Por lo tanto, es apropiado para seleccio-

3 Algunas aclaraciones sobre el número de participantes en la investigación. Por un lado, se tomaron en cuenta casos de trata asistidos por la actual secretaría técnica del comité de Bucaramanga, ya que por medio de esta fue posible la investigación. Por tanto, se entrevistaron personas que participaron en estos casos específicos. Por otro lado, se advierte que en el ámbito público las instituciones rotan sus funcionarios permanentemente, por lo que no fue posible contactar a más personas que hayan participado en estos casos. Por último, no fue posible contactar a más de una víctima por razones logísticas y de seguridad. 
nar sujetos inmersos en unas circunstancias específicas y profundizar en su experiencia.

\section{Recolección de información}

Toda la información fue recolectada a través de entrevistas semiestructuradas. El guión de entrevista fue abierto y flexible para adaptarse a cada entrevistado (Díaz, Torruco, Martinez y Varela, 2013) y se dividió en cuatro momentos: 1. Identificación del entrevistado; 2. Reconstrucción de los casos en los que el participante haya estado involucrado; 3. Balance evaluativo; 4. Cierre. Asimismo, antes de abordar las preguntas, se diligenció un formato de consentimiento informado con los pormenores de la investigación y el tratamiento de la información.

En síntesis, las entrevistas buscaron una reconstrucción de los casos para conocer, a través de la memoria de los entrevistados, cómo y qué servicios de asistencia se prestaron a las víctimas, la identificación de estas y la coordinación interinstitucional. Este ejercicio de entrevista con diversos actores permitió tener una mirada multilateral para conocer el tema. Como aclaración, los nombres de quienes participaron y de las personas mencionadas por ellos han sido cambiados por pseudónimos para proteger su identidad.

Todas las entrevistas fueron grabadas en audio para ser analizadas acorde a los procedimientos propios de la investigación cualitativa, teniendo como brújula el proceso sugerido por la Teoría Fundamentada. Aquél permite clasificar los datos en categorías conceptuales que responden a la pregunta y objetivos de investigación. Lo anterior requirió de la transcripción de todas las entrevistas y su codificación a través de un “(...) proceso analítico por medio del cual se fragmentan, conceptualizan e integran los datos para formar una teoría" (Strauss y Corbin, 2002:11). De esta manera, el trabajo se dividió en dos fases: codificación abierta y codificación axial.

En la codificación abierta “(...) los datos se descomponen en formas discretas, se examinan minuciosamente y se comparan en busca de similitudes y diferencias" (Strauss y Corbin, 2002:111). Para cumplir con esto, la información recabada fue descompuesta hasta la mí- 
nima unidad de análisis cualitativo, representado en este caso en frases o palabras. Todos los datos extraídos de las entrevistas recibieron una etiqueta correspondiente a una acción o idea en concreto relacionada con las categorías de la investigación. De esta forma, toda la información recolectada se clasificó en categorías de análisis iniciales para facilitar una lectura minuciosa.

Posteriormente, toda esta información empieza a juntarse alrededor de unos núcleos comunes a través de la codificación axial, definida por Strauss y Corbin como el "proceso de relacionar las categorías a sus subcategorías, denominado axial porque la codificación ocurre alrededor del eje de una categoría, y enlaza las categorías en cuanto a sus propiedades y dimensiones" (2002:134). Durante esta fase, las categorías y sub-categorías de análisis -etiquetadas durante la codificación abierta- empezaron a relacionarse entre sí construyendo esquemas explicativos para las dinámicas de asistencia a víctimas de trata de personas en Bucaramanga (Strauss y Corbin, 2002).

Para la realización de este análisis se empleó Atlas.ti, un software de investigación diseñado especialmente para el análisis de datos cualitativos.

\section{Resultados y análisis}

En el marco de esta investigación identificación de las víctimas es el proceso mediante el cual el Comité Municipal de Lucha Contra la Trata de Personas de Bucaramanga conoce un posible caso. De lo relatado por los participantes, se desprende que dicha actividad se fundamenta en tres acciones: la recepción del caso por parte del Comité de Bucaramanga, el análisis y la activación de la ruta de asistencia. 
Figura 1: identificación de víctimas de trata de personas en Bucaramanga.



\section{Recepción del caso}

La recepción del caso es el momento en que la institucionalidad conoce la existencia de una presunta víctima. Esto no suele ocurrir de manera activa -a través de acciones, como las redadas, orientadas a rescatar víctimas- sino de formas más reactivas, ya sea porque la víctima o un familiar se acerca a la autoridad, o porque su caso es remitido por alguna institución. En los datos de este trabajo predomina el segundo tipo. La remisión es, sobre todo, un proceso administrativo. El mismo está señalado por la elaboración de un documento: el formato de reporte de caso o, simplemente, el formato, diseñado por el Ministerio del Interior para registrar, reportar e identificar víctimas. Este documento debe ser diligenciado tan pronto se tiene conocimiento de un presunto caso (Ministerio del Interior, s.f.). La remisión de víctimas por formatos queda ejemplificada en fragmentos como el siguiente:

Los formatos, la mayoría, (...) vienen referidos de Bogotá. Yo los recibo, a través del formato es que se analiza la situación, porque a ella no se le hace un interrogatorio antes, no, sino que nos vamos dando cuenta con la información que va suministrando, que es la misma que pide el formato de caso (Marta, Comité Municipal de Lucha Contra la Trata de Personas, 9 de octubre de 2019).

Como la entrevistada dejó entrever, una vez que el formato de reporte de caso ya ha sido diligenciado y entregado al comité, dicho 
documento representa a la víctima. Esto ocurre porque, a través de la información contenida en él, se conoce la situación vivida por aquella y se decide activar -o no- los servicios de asistencia. Por lo tanto, diligenciar, gestionar y remitir este formato ágilmente a las instituciones competentes es un paso clave para identificar y asistir a una víctima de trata de forma oportuna. En este trabajo se identifican dos formas de remisión de presuntas víctimas al Comité Municipal: la que ocurre al interior del sistema para el abordaje de la trata de personas -comité interinstitucional y comités territoriales-, o la que procede desde instituciones externas a dicho sistema. A continuación, se describe cada posibilidad.

Figura 2: recepción de casos por parte del comité de Bucaramanga.

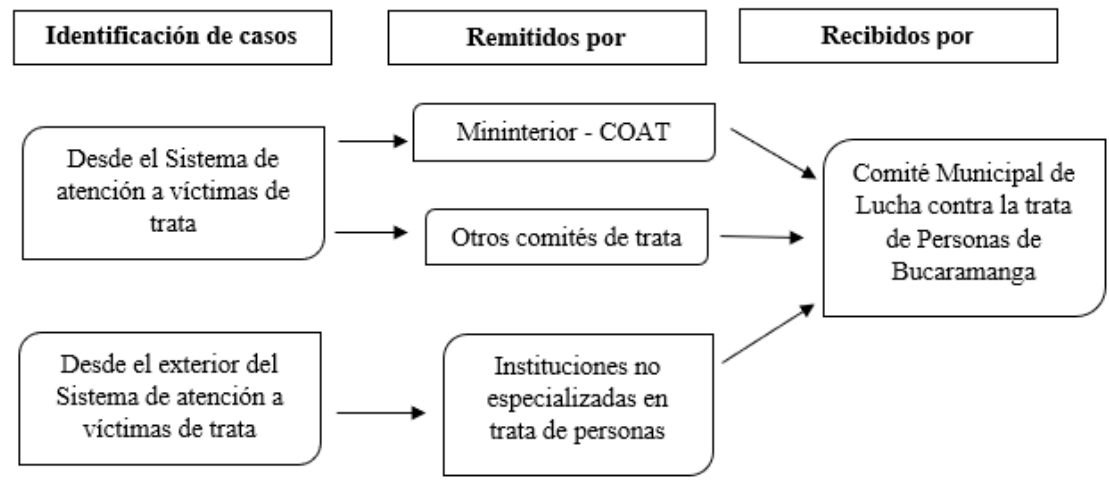

Recepción de casos desde el interior del sistema atención a trata de personas

En este trabajo, dos de los casos fueron identificados por instituciones pertenecientes al sistema de atención en trata de personas. Los mismos corresponden a una víctima de trata interna (Andrés) y una de trata externa (Camila). El primer caso fue remitido por el Comité Distrital de Bogotá, el cual identificó a Andrés y activó ruta de asistencia inmediata. Posteriormente, el formato de caso se remitió a Bucaramanga y fue revisado por el comité junto con la víctima (situación a la que se refiere el fragmento antes citado de Marta). Por otro lado, Camila llegó al Comité Municipal de Bucaramanga luego de que el re- 
lato de su caso pasara por el Ministerio del Interior y el Comité Departamental de Santander. Este caso se conoció gracias a una funcionaria de la Gobernación de Santander (Laura) quien conoció a un familiar de Camila. Al darse por enterada, Laura puso al Comité Departamental de Santander en conocimiento del caso, pero no obtuvo respuesta: “(...) hubo duda si era un caso de trata o no era un caso de trata, ¿sí? Hubo algunas dudas de carácter racional” (Laura, 31 de octubre 2019). En consecuencia, Laura compartió la información con una de sus compañeras de oficina (Mónica) y contactaron al Ministerio del Interior y la Cancillería:

(...) vía el funcionario que maneja el tema de derechos humanos nos hicimos los datos específicos de la persona que maneja lo de trata de personas a nivel de cancillería. Entonces ella nos da la orientación, nos dice 'mándanos por favor este relato...', de hecho nos recuerda que tenía que ser canalizado vía comité y vía los formatos para la presentación de los casos. (...) Entonces ¿qué hacemos nosotros? Con base en la información aportada por la tía, llenamos en líneas generales en qué consistía la situación y lo enviamos (Laura, 7 de noviembre de 2019).

Las funcionarias notificaron el caso a las autoridades nacionales y obtuvieron respuesta del Ministerio del Interior a través del Centro Operativo Anti-trata (en adelante COAT) ${ }^{4}$ :

Ellos generan unas respuestas, y ponen en conocimiento, ahí sí, pues digamos $(. .$.$) a la persona encargada de la secretaría técnica del co-$ mité departamental. Entonces ahí se viene una atención. Se producen respuestas por parte de ese equipo (...) el COAT (Mónica, 7 de noviembre de 2019).

Este caso refleja la importancia que tienen los formatos para que funcione el sistema de asistencia y protección a víctimas. Además,

4 El COAT es parte del Ministerio del Interior y, junto con el Grupo de Lucha Contra la Trata de Personas, el Comité Interinstitucional y los Comités Departamentales coordinan la asistencia a las víctimas del delito en todo el país. 
parece indicar que la activación de la ruta no suele ser tan expedita como la normatividad prescribe, siendo necesaria la insistencia de una persona interesada para que el caso reciba atención. Adicionalmente, pareciera que, a pesar de la descentralización de la política pública anti-trata, es fundamental que una entidad centralizada como el COAT oficie para que a nivel territorial se emprendan acciones. Tal cosa podría ser especialmente cierta para los casos de trata externa que, necesariamente, son gestionados por la Cancillería y el Ministerio de Relaciones Exteriores.

Una vez Camila llega a Bucaramanga y se diligencia el formato de reporte junto con una de las funcionarias de la gobernación, su caso es remitido al Comité Municipal:

(...) yo entrego el formato a UNODC $\mathrm{UN}^{5},(\ldots)$ y no vuelvo a enterarme de ella. Se supone que ella ya está enrutada, ellos la han acogido (...) tengo la idea de que Marta eee, cuando me pide los formatos, que se debe llenar, ella sí explica que la ruta es que el caso será llevado a un comité, que eso va a ser analizado en comité (Mónica, 7 de noviembre de 2019).

La remisión del caso al comité de Bucaramanga está representada en la acción "entregar el formato". En este sentido, la recepción de un caso puede describirse como un proceso administrativo y de "papeleo" en tanto no se concreta ninguna acción de asistencia hasta que los respectivos formatos sean diligenciados y revisados. Tal cosa deja a la presunta víctima en situación de suspenso: su asistencia y protección depende de la agilidad institucional para diligenciar estos formatos.

En conclusión, en la remisión de casos dentro del sistema, cuando un caso de trata de personas llega al comité de Bucaramanga remitido desde otro comité o institución encargada de abordar estos casos, su llegada se representa en la entrega del reporte de caso a la secretaría técnica; de igual forma el diligenciamiento del reporte de caso es una acción clave, ya que a partir de este documento las instituciones cono- 
cen estos casos y empiezan a actuar. Finalmente, se encontraron indicios de desconocimiento del delito de trata de personas en funcionarios públicos encargados de asistir víctimas, lo cual puede llevar a que casos como el de Camila no sean identificados por la institucionalidad de forma oportuna.

Casos recibidos desde fuera del sistema de atención

La segunda forma de remisión es externa al sistema. Este fue el caso de Daniela, quien fue a un consultorio jurídico a recibir orientaciones y se le recomendó ir al comité municipal de Bucaramanga: “(...) se fue entonces para una cosa jurídica, un consultorio jurídico, y de allá fue que le dijeron que eso era trata de personas, que por qué no venía acá a la alcaldía" (Marta, 9 de octubre de 2019). De esta forma, una institución externa al sistema conectó a una presunta víctima de trata con las instituciones competentes. Sin embargo, Daniela tuvo un primer contacto con otra institución que sí hace parte del Comité Municipal de Bucaramanga: "Estuve hablando con la Defensoría del Pueblo pero (...) no, no volví más. (...) uno va y se da cuenta cuando a la persona le interesa el problema de otra persona" (Daniela, 20 de diciembre de 2019). Así, la víctima manifestó que la institucionalidad no mostró interés en su caso, lo cual la llevó a decidir no volver. De esta forma, terminó siendo reconocida y remitida al Comité por una institución sin competencias legales para el abordaje de la trata de personas. En este caso, como en el de Camila, se aprecia que la identificación de las víctimas puede ocurrir solo después de algún intento fallido. No se trata pues de un procedimiento que el comité o sus miembros individualmente considerados realicen con facilidad.

La situación vivida por Daniela también ejemplifica otra cuestión: la reacción de los funcionarios encargados cuando no existe formato de reporte. En este escenario, la propia secretaría técnica del comité de Bucaramanga se encargó de entrevistar a la persona y llenar el formato: "Ella vino acá, me expuso acá su caso (...) Ella la tengo yo actualmente" (Marta, 9 de octubre de 2019). Sobre este encuentro, Daniela comentó que: “(...) ella me entrevistó. Siempre duramos como, no sé, 
un rato hablando. Y yo le comenté paso a paso lo que había sucedido. Cómo habían sucedido las cosas" (Daniela, 20 de diciembre de 2019).

Es interesante analizar la diferencia que existe en la relación del comité de Bucaramanga con una víctima cuando es remitida desde el sistema de atención a trata de personas o una institución externa al mismo. En el primer escenario, debido a que la remisión se condensa en un reporte de caso diligenciado, este documento es la principal guía del comité para conocer a la víctima y tomar decisiones sobre su caso. Por otro lado, cuando la víctima no ha sido referida por una institución competente en trata $-y$, por tanto, no existe un formato de reporte- el contacto entre la secretaría técnica del comité y la víctima es personal.

En conclusión, de la recepción de casos en el comité de Bucaramanga hay varias particularidades por mencionar. Por un lado, ninguno tuvo como primer contacto directo a un miembro del comité municipal: dos fueron referidos desde comités de otra ciudad o nivel territorial y uno fue remitido por una institución externa al sistema. Así, vale la pena indagar sobre el contacto y cobertura que el comité de Bucaramanga tiene con la realidad de su territorio, ya que para la recepción de presuntos casos han sido necesarios múltiples pasos administrativos que finalmente llevan al reconocimiento de aquellos. La seguridad de una presunta víctima puede estar condicionada por estas dinámicas que, en ocasiones, retrasan la atención y puede motivarla a desistir de sus intentos para ser atendida.

Por otro lado, llama la atención que en las respuestas de los funcionarios públicos no haya referencias a las acciones y/o declaraciones de las víctimas. Casi todo el contenido de las entrevistas resalta labores institucionales o del personal técnico. Solo hay una frase que alude directamente a una de las víctimas y es su participación para llenar el formato de reporte de caso, como se menciona en la primera cita de la entrevista a Marta. Esto puede indicar una percepción de las víctimas como un sujeto pasivo durante la asistencia. También, llama la atención que el Comité Municipal de Bucaramanga ha sido dependiente de instituciones externas para conocer presuntos casos. Esto sugiere la necesidad de fortalecer su comunicación y colaboración con entidades que puedan tener conocimiento de víctimas, como verbigracia, con- 
sultorios jurídicos, instituciones de salud privadas, organizaciones no gubernamentales, etc.

\section{Análisis de caso}

El análisis del caso es la revisión que el comité realiza del reporte y determina si la experiencia de la persona coincide con el tipo penal de trata de personas para, posteriormente, activar la ruta de atención. En los datos, se aprecia que el comité de trata de personas de Bucaramanga tiene, al menos, tres formas de analizar un caso: el análisis autónomo, exclusivo de la secretaría técnica; el análisis colectivo, con participación de las instituciones del comité; y el análisis con la víctima.

Tabla 3: Análisis de reportes de casos de trata de personas

\begin{tabular}{lll}
\hline $\begin{array}{c}\text { Tipo de } \\
\text { análisis }\end{array}$ & \multicolumn{1}{c}{ Descripción } & \multicolumn{1}{c}{ Condiciones } \\
\hline Autónomo & $\begin{array}{l}\text { La secretaría técnica del Comité se } \\
\text { encarga de revisar el presunto caso. }\end{array}$ & $\begin{array}{l}\mathrm{Si} \text { el relato del caso es lo } \\
\text { suficientemente claro, la secretaría } \\
\text { técnica procede a activar la ruta de } \\
\text { asistencia. }\end{array}$ \\
Colectivo & $\begin{array}{l}\text { Se convoca a todo el Comité a revisar } \\
\text { un presunto caso de trata de personas y }\end{array}$ & $\begin{array}{l}\text { Si hay poca claridad sobre el caso } \\
\text { se convoca a todo el comité para } \\
\text { revisar y analizarlo conjuntamente. }\end{array}$ \\
Con la & $\begin{array}{l}\text { La secretaría técnica del Comité, junto } \\
\text { con la víctima, revisa el reporte de caso }\end{array}$ & $\begin{array}{l}\text { Casos en los cuáles ya ha sido } \\
\text { identificada, asistida y remitida por } \\
\text { otro comité de trata de personas. }\end{array}$ \\
& $\begin{array}{l}\text { para validar la información y conocer } \\
\text { los detalles. }\end{array}$ & \\
\hline
\end{tabular}

En este aspecto, proceder de una u otra forma depende del criterio interpretativo de la secretaría técnica, instancia que decide si tipifica y activa la ruta de asistencia autónomamente, si es necesario convocar al comité para un análisis colectivo, o si entrevista a la víctima. Así, la primera vía es la que determina a las otras dos:

(...) cuando hay como, las situaciones que le pasaron a la víctima son claras, a modo de ver de la secretaría técnica, que sí es una posible víctima, pues, automáticamente se activa la ruta. (...) por lo contrario, si no tenemos claro y estamos en duda, y tenemos algunas diferencias entre los que analizamos el caso, convocamos a comité 
extraordinario para que todo el comité se responsabilice del caso y se opte por decir 'sí la vamos a tipificar, sí le vamos a prestar la atención mediata o si nos corresponde atención inmediata' (Marta, 9 de octubre de 2019).

Aquí, se aprecia que el análisis de un caso de manera colectiva o individual está relacionado con la dificultad de tipificar el relato. Es decir, el comité actúa según una lógica práctica que responde a cada caso: de acuerdo con la claridad que tenga sobre la situación decide actuar como conjunto o seguir las directrices de la secretaría técnica. Aunque no es mencionado textualmente, se intuye en el fragmento anterior que la claridad de un caso hace referencia a las coincidencias entre el relato de una víctima sobre su experiencia y el tipo penal de la trata de personas. Esto puede dificultar la identificación de presuntas víctimas, ya que no necesariamente hay una metodología clara para realizar dicha tipificación.

Como ya se mencionó, de los tres casos reconstruidos aquí solo uno fue identificado por el comité de Bucaramanga. En dicho caso, la representante de la secretaría técnica mencionó haberse reunido con la víctima, pero no pudo recordar si realizó convocatoria para análisis colectivo: “(...) yo creo que convoqué a comité. Yo creo que sí, hicimos comité, no me acuerdo. La verdad no me acuerdo" (Marta, 9 de octubre de 2019). Por lo anterior, aunque la entrevistada describió cómo se analiza -normalmente- un reporte, esta información no pudo ser contrastada con la realidad de este caso.

Por último, como fue mencionado en el apartado de recepción del caso, el análisis del reporte proveniente de Bogotá consistió en revisar, junto con Andrés, el formato recibido desde el comité distrital para la confirmación de la información contenida en dicho formato. En este sentido, cuando una víctima ya ha sido identificada y asistida por otro comité antes de ser remitido al de Bucaramanga, este último puede analizar el reporte de caso, junto con la víctima, para conocer cuál fue su situación, hacer reconocimiento de sus necesidades y continuar con los servicios de asistencia.

Escoger una de estas formas de análisis depende de las condiciones que presente el caso y de la forma como la secretaría técnica decida 
responder a ellas. La información recolectada indica que el comité de Bucaramanga tiende a delegar la responsabilidad de la revisión a su secretaría técnica y su criterio interpretativo. Tal cosa requiere de experticia jurídica para una apropiada lectura de los relatos de las víctimas a la luz de la normatividad. A su vez, en los casos donde hay entrevista personal entre la secretaría técnica y una presunta víctima también pueden ser necesarias habilidades interpersonales en términos de sensibilidad, empatía y atención en crisis. De lo anterior se desprende la necesidad de que quienes ocupen las secretarías técnicas tengan una formación amplia que integre distintas áreas de conocimiento administrativo, jurídico y psico-social. De manera alternativa, la secretaría técnica debería reposar sobre un equipo con capacidades instaladas en materia jurídica y psico-social.

\section{Activación de la ruta de asistencia}

Ya que la activación de la ruta de asistencia es dependiente y casi simultánea al análisis del caso, las dinámicas de esta activación son, en la práctica, inescindibles del reporte. Una vez que se decide activar la ruta, la secretaría técnica contacta con las instituciones competentes para gestionar los servicios comprendidos en la normativa. Esto se hace oficiando, es decir, enviando solicitudes escritas a otros funcionarios. Una de las entrevistadas describe así dicha acción: “(...) Le escribí a la policía, le escribí a la defensoría del pueblo, y le escribí a la fiscalía general de la nación. Y le remití a la oficina de la mujer" (Marta, 9 de octubre de 2019). Es claro que esta labor está relacionada con las dinámicas operacionales al interior de la secretaría técnica y el nivel de comunicación/coordinación que esta tiene con las demás instituciones.

A partir de toda la información analizada, se puede concluir que la identificación de víctimas por parte del Comité de Bucaramanga es un trabajo de construcción administrativa de un caso a partir del estudio de la situación vivida por la presunta víctima y mediante el uso del formato de reporte y, en algunos casos, el relato de la propia víctima. Una vez que se ha construido el caso, a partir de la normativa, las instituciones competentes determinan si las circunstancias corresponden con el tipo penal de trata de personas. Estas actividades están parcialmente 
centralizadas en la secretaría técnica del comité que, de forma autónoma, puede determinar si es necesario convocar a otros o si se puede desestimar el caso. Esta decisión está basada en la claridad o duda que genere el reporte entre quienes lo analizan. Una vez que se reconoce la trata de personas, de inmediato se activa la ruta de asistencia mediante notificación de la secretaría técnica del comité a las instituciones competentes para prestar los servicios de atención que se requieran.

\section{Conclusiones}

El Comité Municipal de Lucha contra la Trata de Personas de Bucaramanga fue creado en el año 2010 atendiendo al mandato de la Ley 985 de 2005 (art 14), con el objetivo de hacer frente a este delito a nivel local. Asimismo, para cumplir con esta labor, el Comité se rige por la normativa nacional y las directrices internacionales (Decreto 1066 de 2015, Protocolo de Palermo). Este documento tiene como objetivo compartir los resultados obtenidos en la indagación sobre los procesos de identificación de víctimas de trata en Bucaramanga.

A partir de lo anterior, se ha descrito la identificación de víctimas principalmente como una labor administrativa de construcción del caso: el papeleo o las gestiones institucionales relacionadas con el diligenciamiento y entrega del formato de reporte de caso, lo cual valida la recepción de una víctima por parte del Comité y permite dar inicio a la asistencia, son las actividades principales de esta identificación. Por ello, tal vez contrario a lo que se pueda imaginar, la asistencia a víctimas de este delito se parece más a un trámite burocrático-administrativo que a un contacto directo entre las víctimas y las instituciones encargadas de la asistencia. Asimismo, esta construcción administrativa del caso ha estado centralizada en las labores de la secretaría técnica del comité. Por último, se ha evidenciado que el comité puede tener dificultades a la hora de identificar casos de trata a nivel local.

Por lo anterior, es importante que el Comité disponga de canales de recepción e identificación de casos apropiados. Para ello, se recomienda preparar a todas las instituciones en la identificación de casos de trata, especialmente aquellas cuyas responsabilidades institucionales les facilite el contacto con presuntas víctimas. Estas pueden ser la 
Policía Metropolitana de Bucaramanga, la Defensoría del Pueblo y el Instituto Colombiano de Bienestar Familiar, así como instituciones de servicios de salud contactadas a través de la Secretaría de Salud Municipal. Así mismo, en cuanto al análisis de cada caso, en este trabajo se han encontrado, por lo menos, tres formas en que el comité lo ha hecho. Es recomendable que se estandarice una sola forma de análisis de caso, preferiblemente involucrando al comité en su totalidad, ya que esto permite tener distintas perspectivas sobre el relato de las presuntas víctimas.

Por último, cabe recordar que esta investigación solo pudo reconstruir tres casos de trata de personas en Bucaramanga, por tanto, es pertinente seguir investigando y profundizando sobre este tema, de manera que los resultados aquí expuestos sean ampliados y debidamente contrastados.

\section{Referencias bibliográficas}

Aceros, Juan Carlos; Vargas, Jackeline, y Reyes, Johana (2017). Trayectorias territoriales de la asistencia a víctimas de trata de personas. Análisis cualitativo desde la perspectiva de los actores institucionales en Santander. Revista criminalidad, 59(2), 33-48. Recuperado de: http://www.scielo.org.co/pdf/crim/v59n2/1794-3108crim-59-02-00033.pdf

Alarcón, Nancy. (2016). Escenario para la protección y asistencia a víctimas de trata de personas en Colombia. Derecho y realidad, 14(28), 155-180.

Chetty, Sylvie (1996). The Case Study Method for Research in Small-and Medium-Sized firms. International small business journal, 73-85. DOI: https://doi. org/10.1177/0266242696151005

Department of State (2020). Human Trafficking report. Washington D.C., EEUU.

Diaz, Laura; Torruco, Uri; Martinez, Mildred y Varela, Margarita (2013). La entrevista, recurso flexible y dinámico. Investigación en Educación media, 2(7), 162-167. Recuperado de: https://www.redalyc.org/pdf/3497/349733228009.pdf

Glaser, Barney y Strauss, Anselm (1967). The discovery of grounded theory: Strategies for qualitative research. AldineTransaction.

Halaweh, Mohana; Fidler, Christine; y McRobb, Steve (2008). Integrating the Grounded Theory Method and Case Study Research Methodology Within IS Research: A Possible "Road Map". International Conference on Information Systems (ICIS). Recuperado de: https://aisel.aisnet.org/cgi/viewcontent.cgi?article=1052\&context=icis 2008

Hernandez, Roberto; Fernandez, Carlos y Baptista, Pilar (2010). Metodología de la investigación. México D.F., México, McGrawHill.

Ministerio del Interior (s.f.). Dirección de Gobierno y Gestión Territorial. Lucha contra la trata de personas. Programa de protección y asistencia. Recuperado de: 
https://colaboracion.dnp.gov.co/CDT/CajaHerramientas/Caja PISCC/Proyectos $\% 20 \mathrm{y} \% 20$ Financiacion/Programas $\% 20$ Nacionales/Ministerio $\% 20 \mathrm{del} \% 20$ Interior/Trata\%20de\%20personas/Presentacion DecretoTrata.pdf

Naciones Unidas (2000). Protocolo para prevenir, reprimir y sancionar la trata de personas, especialmente mujeres y niños, que complementa la Convención de las Naciones Unidas contra la Delincuencia Organizada Transnacional. Palermo, Italia.

Organización Internacional de Migraciones (2020). Counter Trafficking Data Collaborative. Recuperado de: https://www.ctdatacollaborative.org/dashboard/global-ctdc-dashboard

San Martin, Daniel (2014). Teoría fundamentada y Atlas.ti: recursos metodológicos para la investigación educativa. Revista electrónica de investigación educativa. 16(1), 104-122. Recuperado de: https://redie.uabc.mx/redie/article/view/727/891

Strauss, Anselm y Corbin, Juliet (2002). Bases de la investigación cualitativa. Técnicas y procedimientos para desarrollar la teoría fundamentada. Medellín, Colombia, Editorial Universidad de Antioquia.

United Nations Office on Drugs and Crime (2018). Global report on trafficking in persons. United Nations publication.

Yin, Robert (2014). Case Study Research - Design and Methods, Applied Social Research Methods. California, Estados Unidos, SAGE publications.

Recibido: 31/07/2021

Aceptado: 13/10/2021 